\title{
Emission of high energy particles in heavy ion collisions and a non-equilibrium equation of state
}

\author{
Alexander T. D'yachenko ${ }^{1, *}$ and Ivan A. Mitropolsky ${ }^{2, * *}$ \\ ${ }^{1}$ Emperor Alexander I Petersburg State Transport University, 9 Moscow ave., St.Petersburg, 190031 \\ Russia \\ ${ }^{2}$ NRC "Kurchatov Institute", B.P. Konstantinov Petersburg Nuclear Physics Institute, Gatchina, 188300 \\ Russia
}

\begin{abstract}
A hydrodynamic approach with a non-equilibrium equation of state is used to describe the collisions of heavy ions at medium and intermediate energies. In the development of this approach, with the inclusion of nuclear viscosity effects and the introduction of an amendment to the microcanonical distribution, the double differential cross sections of proton emission in collisions of different nuclei are calculated, which are in agreement with the available experimental data on the emission of high-energy particles, including the cumulative spectral region.
\end{abstract}

\section{Introduction}

Along with molecular dynamics and the solution of the dynamic Boltzmann equation, nuclear hydrodynamics is an effective method for describing the dynamics of the interaction of heavy ions at medium and intermediate energies (see, for example, [1]). As a rule, the equilibrium equation of state (EOS) is used, which assumes the establishment of a local thermodynamic equilibrium in the system. At high energies, a hybrid model was proposed in [2, 3], which includes a fast non-equilibrium stage and the subsequent description of the dynamics of the nucleus - nucleus interaction based on the hydrodynamics of an ideal fluid. In our papers, the dynamics of heavy ion collisions involves the use of a non-equilibrium equation of state [4-7] taking into account the effects of nuclear viscosity. This allowed describing energy spectra of protons, pions, and fragments for the collisions of different nuclei at the medium-energy range [5-7]. However, "high-energy tails " of proton spectra obtained in [8], as it turned out, are not reproduced near the kinematic limit of the spectrum. They turn out more harden when we use a grand canonical ensemble for the distribution function of emitted protons. In this paper, we have succeeded to introduce an amendment with account of the microcanonical distribution in the high-energy part of the spectrum to reproduce experimental data [8] without breaking the agreement with other data. In addition, we have succeeded to reproduce the high-momentum proton distributions for the ${ }^{12} \mathrm{C}+{ }^{9} \mathrm{Be} \rightarrow \mathrm{p}+\mathrm{X}$ reaction at an ${ }^{12} \mathrm{C}$ ion energy of 300,600 and $950 \mathrm{MeV} /$ nucleon at the angle of $3.5^{\circ}$ [9], which are related to the cumulative region and are not quite well described by the "molecular dynamics" and other cascade models.

\footnotetext{
*e-mail: dyachenko_a@mail.ru

**e-mail: mitropolsky_ia@nrcki.pnpi.spb.ru
} 


\section{The model}

To describe the collisions of heavy ions, we use the non-equilibrium hydrodynamic approach [4-7], in which the kinetic equation for the nucleon distribution function $f(\vec{r}, \vec{p}, t)$ is solved jointly with the equations of hydrodynamics, which are essentially local laws of conservation of mass, momentum and energy. This leads to the need of using the non-equilibrium equation of state, the pressure tensor in which can be written as $[6,7]\left(\delta_{i j}\right.$ is a Kronecker symbol, $i, j=1,2,3)$

$$
P_{i j}=P_{(k i n, 1) i j} q+\left(P_{(k i n, 0)}(1-q)+P_{i n t}\right) \delta_{i j},
$$

where $P_{(k i n, 1) i j}$ is the non-equilibrium anisotropic pressure tensor corresponding to the deformed Fermi surface, $P_{(k i n, 0)} \delta_{i j}$ is the isotropic pressure tensor corresponding to a local thermodynamic equilibrium, $q(\vec{r}, t)(0<q<1)$ is the relaxation factor found from the kinetic equation by taking the moment determining the degree of anisotropy of the distribution function in the momentum space [6, 7]. The kinetic terms $P_{(k i n, 1) i j}$ and $P_{(k i n, 0)}$ included in pressure and kinetic term $e_{k i n}$ included in energy density $e\left(e=e_{k i n}+e_{i n t}\right)$ are determined by the thermal and Fermi motion of the nucleons.The interaction terms $P_{i n t}$ and $e_{i n t}$ correspond to the self-consistent mean field with parameters determinable by assignment of the binding energy $E_{b}=-16 \mathrm{MeV}$, of the normal density $\rho_{0}=0.145 \mathrm{fm}^{-3}$ and of the compression module $K=210 \mathrm{MeV}$ for the cold nuclear matter.

After selecting the local heating region, hot spot is the overlap region of the colliding nuclei, we analyze the stages of compression, expansion and freeze-out of the nuclear matter during the collision of heavy ions. At the compression stage, the shock wave with a changing front is formed [5]. At the expansion stage [5-7], when the shock wave reaches the boundaries of hot spot, the initially compressed system is expanded.We describe it in the relaxation approximation taking into account the nuclear viscosity [10]. For the relaxation time we take $\tau=\lambda / v_{T}$, where $\lambda=1 / \sigma \rho$ is the mean free path, $\sigma \approx 40 \mathrm{mb}$ is the total nucleonnucleon cross section, $\rho$ is the nucleon density, and $v_{T}$ is the average velocity of the thermal motion of the nucleons. At the freeze-out stage, when the system reaches a critical density $\rho^{*}\left(d P_{\text {int }}(\rho) /\left.d \rho\right|_{\rho=\rho^{*}}=0\right)$ also called the freeze-out density, the system is not able to "hold itself" and the secondary particles are formed [4-7, 10,11]. As a result, the double differential cross-section of proton emission has the following form (where $b$ is the impact parameter, $\hbar$ is a Planck constant, $\vec{r}$ is the radius vector):

$$
\frac{d^{2} \sigma}{d E d \Omega}=\frac{2 \pi}{(2 \pi \hbar)^{3}} \int G(b) b d b \int d \vec{r} \gamma(E-\vec{p} \vec{v}) p f(\vec{r}, \vec{p}, t),
$$

where the distribution function of the emitted protons without a negligible non-equilibrium component at the freeze-out stage is equal to

$$
f(\vec{r}, \vec{p}, t)=g\left[\exp \left(\frac{\gamma(E-\vec{p} \vec{v})-\mu+T \delta}{T}\right)+1\right]^{-1},
$$

where the spin factor $g=2, \Omega$ is the solid angle, $E=\sqrt{p^{2}+m^{2}}, \gamma\left(\gamma=1 / \sqrt{1-v^{2}}\right)$ and $\vec{p}$ are respectively the total energy, the Lorentz factor and the proton momentum; $\vec{v}(\vec{r}, t)$ is the velocity field at the freeze-out stage found from solving hydrodynamic equations for a moving hot spot $[5,7,10], G(b)$ is the factor which reflects that the cross section of the hot spot formation is always greater than the geometric one $\left(G(b)=\sigma_{t} / \sigma_{0}\right.$, where $\sigma_{t}=\pi\left\langle R_{b}\right\rangle^{2}$ is the total cross section for the formation of the hot spot, and $\left\langle R_{b}\right\rangle$ is the average radius of interaction of the overlapping parts of the colliding nuclei, $\sigma_{0}$ is the geometric section of the overlapping parts), $\mu\left(\mu=\mu_{T}+m\right)$ is the chemical potential, which is found from the 
conservation of the average number of particles for a grand canonical ensemble, $T$ is the temperature at the freeze-out stage, $\delta$ is correction for the microcanonical distribution, which for the kinetic energy $\epsilon=E-m>E_{1}$ is equal to

$$
\delta=\left[-M \ln \left(1-\frac{\gamma(E-\vec{p} \vec{v})-m}{M T}\right)-\frac{\gamma(E-\vec{p} \vec{v})-m}{T}\right],
$$

where $M=3 / 2 N, N$ is the number of nucleons in the thermostat, $E_{1}\left(E_{1}>>T\right)$ is the energy that is close to the energy of the thermostat, i.e. close to the kinematic limit for the energy of the system. We have also chosen the energy value $E_{2}\left(E_{2}<E_{1}\right)$, when the distribution function decreases by an order of magnitude compared to its maximum. When $\epsilon<E_{2}$ the amendment $\delta$ was supposed to be equal to zero. In the energy interval $E_{1}>\epsilon>E_{2}$ it was a linear interpolation between zero and expression (4). Here the correction $\delta$ is found for the Boltzmann limit of an ideal gas, since deviations from a grand canonical distribution of the Fermi gas are manifested on the "tails" of the energy spectra, when the Fermi distribution coincides with the Boltzmann limit.

The probability of a microcanonical distribution in the limit of the Boltzmann limit of an ideal gas is (for details, see Appendix) as follows:

$$
W_{\epsilon}=C_{M}\left(1-\frac{\epsilon}{E_{0}}\right)^{M}=C_{M} \exp \left(M \ln \left(1-\frac{\epsilon}{E_{0}}\right)\right),
$$

where $\epsilon$ is the kinetic energy of the system, $E_{0}=M T$ is the energy of the thermostat, $C_{M}$ is the normalization factor $[12,13]$. As a result, in the limit of a large number of particles $N$ at $M=3 / 2 N \rightarrow \infty$, expression (5) becomes a canonical distribution:

$$
W_{0 \epsilon}=C_{M} \exp \left(-\frac{\epsilon}{T}\right)
$$

Thus, on the tails of the energy distributions, using formula (4), we find the amendment for the microcanonical distribution (3), which changes the usual Fermi-Dirac distribution describing the system well away from the tails of the proton spectra. Moreover, in formulae (3)-(4) it is taken into account that the energy of the system is recalculated in accordance with the Lorentz transformations. The energy in the distribution (3) is reckoned from the value of the self-consistent mean field with allowance for the nuclear surface energy, since the nucleons are "locked" by the mean field.

In addition to the contribution of (2) to the cross section for the emission of protons from the hot spot, we have also taken into account the contribution from the fusion of the nonoverlapping parts of the colliding nuclei so called "spectators".

\section{A comparison with experimental data}

A comparison of the calculated energy spectra of protons emitted in the reaction ${ }^{16} \mathrm{O}+{ }^{27} \mathrm{Al} \rightarrow \mathrm{p}+\mathrm{X}$ at an angle of $30^{\circ}$ with the ${ }^{16} \mathrm{O}$ ion energy of $207 \mathrm{MeV}(\sim 12 \mathrm{MeV} /$ nucleon $)$ (curve 1) and $310 \mathrm{MeV}$ ( $19 \mathrm{MeV} /$ nucleon) (curve 2) with experimental data is shown in Fig. 1. Our calculation describes the data [8] (which have so far been unexplained) with allowance for the introduced amendment for the microcanonical distribution. Without taking this correction into account, the spectra turn out to be more harden (corresponding to this variant of the calculation, the dashed curves la and 2 a go above the points). The agreement with the experiment is achieved for a non-equilibrium equation of state with compression modulus of $K=210 \mathrm{MeV}$ and without breaking the agreement with other data. 


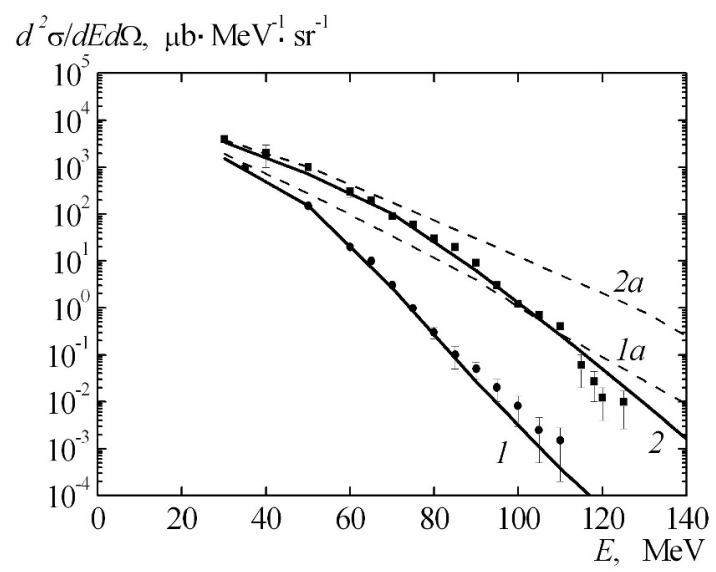

Figure 1. The energy spectra of the protons emitted in the reaction ${ }^{16} \mathrm{O}+{ }^{27} \mathrm{Al} \rightarrow \mathrm{p}+\mathrm{X}$ at an angle of $30^{\circ}$ with the ${ }^{16} \mathrm{O}$ ion energy of $207 \mathrm{MeV}$ (curve 1) and $310 \mathrm{MeV}$ (curve 2). The calculated spectra are shown by solid lines and experimental data [8] are shown by points. The dashed curves la and 2a correspond to calculations without taking into account an amendment for the microcanonical distribution. In the first case, the average temperature of the hot spot $\left\langle T_{h}>\right.$ is equal to $8.3 \mathrm{MeV}$, the chemical potential $<\mu_{T}>$ is equal to $32.9 \mathrm{MeV}$, in the second case, respectively, $<T_{h}>\approx 10 \mathrm{MeV},<\mu_{T}>\approx 31.2 \mathrm{MeV}$, the average radius of the hot spot $<R_{h}>\approx 2 \mathrm{fm}$

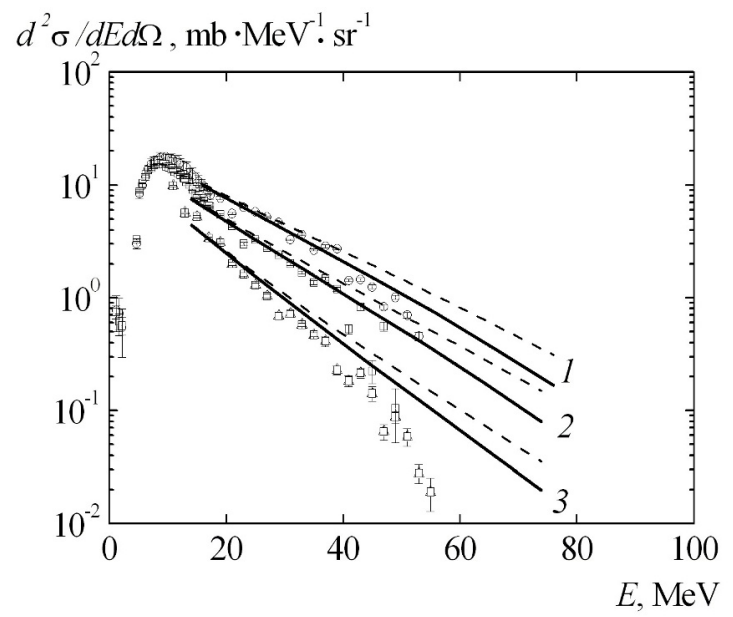

Figure 2. The energy spectra of protons emitted in the reaction ${ }^{14} \mathrm{~N}+{ }^{124} \mathrm{Sn} \rightarrow \mathrm{p}+\mathrm{X}$ calculated with a non-equilibrium equation of state (solid lines) and experimental data (points from [14]) with the ${ }^{14} \mathrm{~N}$ ion energy of $32 \mathrm{MeV} /$ nucleon at an angle of $45^{\circ}$ (curve 1), $62^{\circ}$ (curve 2) and $90^{\circ}$ (curve 3 ). In this case, $<T_{h}>\approx 11.4 \mathrm{MeV},<\mu_{T}>\approx 30 \mathrm{MeV},<R_{h}>\approx 3 \mathrm{fm}$. The dashed curves correspond to the calculations for the traditional equilibrium equation of state. In this case, relaxation factor $q=0$ 


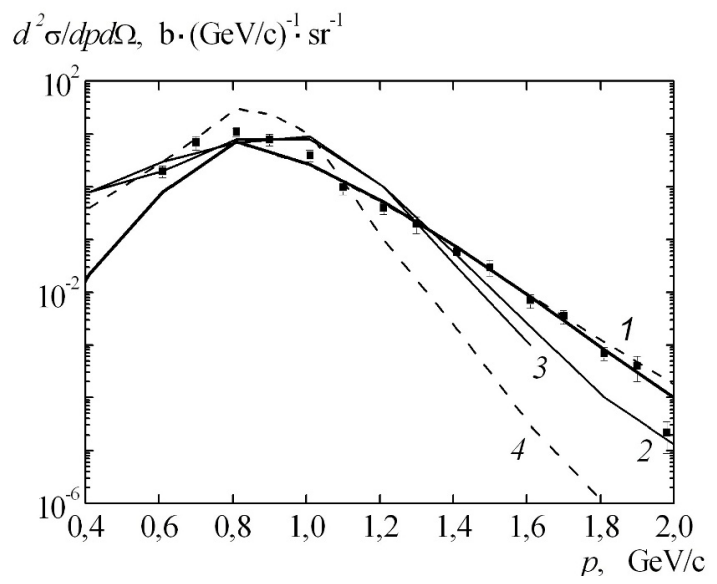

Figure 3. The momentum distributions of protons emitted in the reaction ${ }^{12} \mathrm{C}+{ }^{9} \mathrm{Be} \rightarrow \mathrm{p}+\mathrm{X}$ at an angle of $3.5^{\circ}$ with the ${ }^{12} \mathrm{C}$ ion energy of $300 \mathrm{MeV} /$ nucleon. Curve 1 is our calculation, the dashed curve 1 is our calculation without taking into account the amendment for the microcanonical distribution, the points are the experimental data from [9]. Curves 2, 3, 4 are results of calculations for transport codes [9]. Curve 2 corresponds to the cascade model [15], curve 3 corresponds to the transport model of quark-gluon strings [16], curve 4 corresponds to the model of quantum molecular dynamics (QMD) built into the GEANT4 package [17]. In this case, $<T_{h}>\approx 33.2 \mathrm{MeV},<\mu_{T}>\approx-2.2 \mathrm{MeV},<R_{h}>\approx 2$ fm

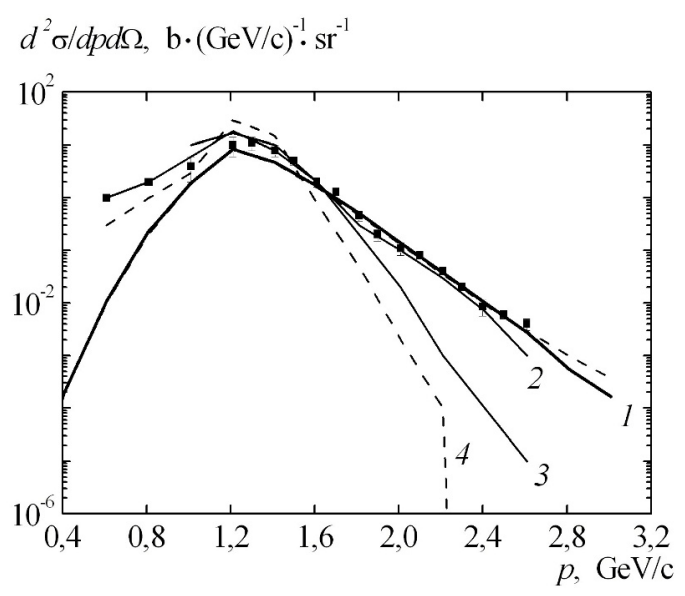

Figure 4. The same that in Fig. 3 with the ${ }^{12} \mathrm{C}$ ion energy of $600 \mathrm{MeV} /$ nucleon, and $\left\langle T_{h}>\approx 42.5\right.$ $\mathrm{MeV},<\mu_{T}>\approx-17.7 \mathrm{MeV}$

In Fig. 2 there are energy spectra of the protons emitted in the reaction ${ }^{14} \mathrm{~N}+{ }^{124} \mathrm{Sn} \rightarrow$ $\mathrm{p}+\mathrm{X}$ at angles of $45^{\circ}$ (curve 1), $62^{\circ}$ (curve 2) and $90^{\circ}$ (curve 3) with the ${ }^{14} \mathrm{~N}$ ion energy of $32 \mathrm{MeV} /$ nucleon. The calculated curves corresponding to the non-equilibrium equation of state (solid lines) agree with the experimental data [14], in contrast to the dashed lines corresponding to the choice of the equilibrium equation of state with $q=0$ which go above the experimental points. At the tails the experimental spectrum is somewhat "cut off," which is 


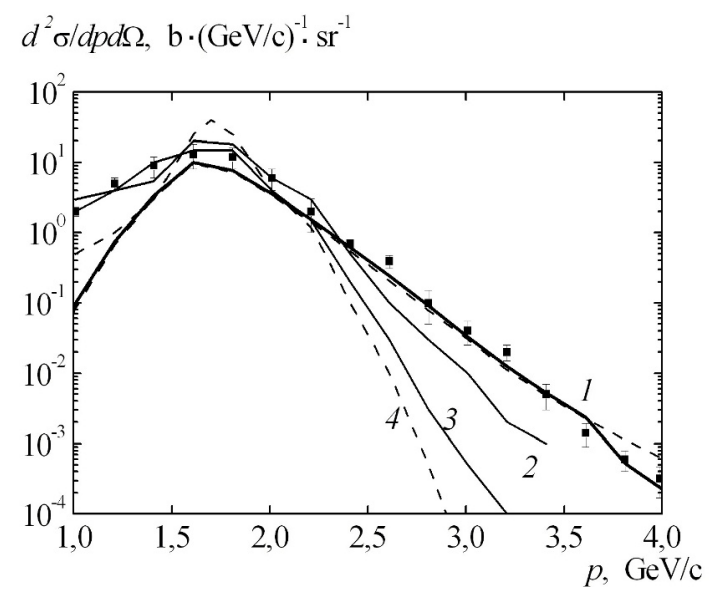

Figure 5. The same as in Fig. 3 with the ${ }^{12} \mathrm{C}$ ion energy of $950 \mathrm{MeV} /$ nucleon, and $<T_{h}>\approx 51 \mathrm{MeV}$, $<\mu_{T}>\approx-37 \mathrm{MeV}$

apparently due to systematic experimental errors and is not reproduced in the calculation. The amendment for the microcanonical distribution is in a significantly higher region of proton energies (see the previous Fig. 1).

Figs. 3-5 show the momentum spectra of protons emitted in the reaction ${ }^{12} \mathrm{C}+{ }^{9} \mathrm{Be} \rightarrow \mathrm{p}+\mathrm{X}$ at an angle of $3.5^{\circ}$ with the ${ }^{12} \mathrm{C}$ ion energy of $300 \mathrm{MeV} /$ nucleon (Fig. 3), $600 \mathrm{MeV} /$ nucleon (Fig. 4) and $950 \mathrm{MeV} /$ nucleon (Fig. 5). The experimental data [9] are marked by points. The solid curve 1 is our calculation. The dashed curve 1 is our calculation without taking into account the amendment for the microcanonical distribution. The curves 2, 3, 4 are results of calculations for transport codes [9]. The curve 2 corresponds to the cascade model [15], the curve 3 corresponds to the transport model of quark-gluon strings [16], the curve 4 corresponds to the quantum molecular dynamics (QMD) model built into the GEANT4 [17]. As can be seen from these figures, in the cumulative region of the spectrum our calculation turned out to be in agreement with the experimental data [9]. The decline in the cross sections by 5 orders of magnitude is reproduced in our approach not worse than in the Monte Carlo transport codes. Some cascade calculations noticeably underestimate these experimental data in the high-momentum region. However, in the region of small momenta our calculation underestimates the experimental data, which may be due to the contribution of the protons formed as a result of ${ }^{12} \mathrm{C}$ fragmentation. The amendment for the microcanonical distribution (solid curve 1) appears in this case only at the very tails of the high-momentum distributions of the protons

\section{Concluding remarks}

Thus in this paper the idea of using a non-equilibrium equation of state in the hydrodynamic approach to describe the high-momentum proton spectra emitted in heavy-ion collisions over a wide energy range has been further developed.

The experimental shoulder in the cross section for the production of protons in the cumulative region is reproduced by our calculations, and sometimes by cascade models. Perhaps this may be due to the contribution of the rescattering of pions to the cumulative production of protons, considered earlier in [18]. 
Acknowledgement. Authors are grateful to V.V. Vechernin, V.N. Kovalenko, V.Yu. Petrov and M.B. Zhalov for useful discussions and to the referee for his valuable comments.

\section{Appendix}

Let us explain how formulae (3) and (4) were obtained. The probability $W_{n}$ of microcanonical distribution of particles for a state with energy $E_{n}$ is [13] ( $A$ is the constant) as follows:

$$
W_{n}=A \int \delta\left(E_{n}+E^{\prime}-E_{0}\right) d \Gamma^{\prime} .
$$

Integrating the $\delta$ - function on the thermostat energy $E^{\prime}, d \Gamma^{\prime}=\left(d \Gamma^{\prime} / d E^{\prime}\right) d E^{\prime}$, we obtain $W_{n}=A \exp \left(S^{\prime}\right) /\left.\Delta E^{\prime}\right|_{E^{\prime}=E_{0}-E_{n}}$, where $E_{0}$ is a given value of the energy of a closed system (in our case, it is the total energy of hot spot), the entropy is $S^{\prime}=\ln \Delta \Gamma^{\prime}$, the statistical weight $\Delta \Gamma^{\prime}=\left(d \Gamma^{\prime} / d E^{\prime}\right) \Delta E^{\prime}$, and $\left(\Delta E^{\prime} /<E^{\prime}>\right) \sim 1 / \sqrt{N}$ is dispersion. The statistical weight of the thermostat $\Gamma^{\prime}=B_{N} V^{N}\left(2 m E^{\prime}\right)^{3 / 2 N} /(2 \pi \hbar)^{3 N}$, where $B_{N}$ is the numerical coefficient, is found in the limit of the Boltzmann ideal gas, where the phase volume of the thermostat is as the volume of the $3 N$-dimensional ball in the momentum space multiplied by the spatial volume $V^{N}$ for $N$ particles [12]. Since $\left(d \Gamma^{\prime} / d E^{\prime}\right)=B_{N} V^{N} 2 m(3 / 2 N)\left(2 m E^{\prime}\right)^{3 / 2 N-1} /(2 \pi \hbar)^{3 N}$, the result will be:

$$
W_{n}=C_{M} \exp \left(M \ln \left(1-\frac{E_{n}}{M T}\right)\right),
$$

where $C_{M}$ is the normalization constant independent of the energy $E_{n}, M=3 / 2 N$. We have neglected the difference between $M$ and $3 / 2 N-1$ and put for an ideal gas $E_{0}=M T$, where $1 / T=d S^{\prime} / d E^{\prime}$. The rest energy balance is also satisfied, so the rest energy is omitted in the expression for $E_{0}$.

The distribution (8) is transformed for a large number of particles $N$ at $M \rightarrow \infty$ into the canonical distribution $W_{0 n}=C_{M} \exp \left(-\frac{E_{n}}{T}\right)$. Similar to the transition to a grand canonical distribution for the system with a variable number of particles [13] we can write $w_{k}=\exp \left(\frac{\Omega_{k}}{T}+\frac{\mu_{T} n_{k}}{T}+M \ln \left(1-\frac{\epsilon_{k} n_{k}}{M T}\right)\right)$, where the chemical potential $\mu_{T}=-T\left(\frac{\partial S}{\partial N}\right)_{E, V}$, and find the thermodynamic potential $\Omega_{k}$ from the normalization conditions. According to Pauli's principle for the fermion occupation numbers we assume $n_{k}=0,1$ and the energies of particles are equal to $E_{k}=\epsilon_{k} n_{k}$. The average number of particles in the k-th state

$$
\begin{aligned}
<n_{k}> & =\frac{\sum_{n_{k}} w_{k} n_{k}}{\sum_{n_{k}} w_{k}}=\frac{\exp \left(\frac{\mu_{T}}{T}+M \ln \left(1-\epsilon_{k} / M T\right)\right)}{1+\exp \left(\frac{\mu_{T}}{T}+M \ln \left(1-\epsilon_{k} / M T\right)\right)} \\
& =\left[1+\exp \left(-\frac{\mu_{T}}{T}-M \ln \left(1-\frac{\epsilon_{k}}{M T}\right)\right)\right]^{-1}
\end{aligned}
$$

determines the distribution function $f=<n_{k}>$ for Fermi gas particles in the quasi-classical state with momentum $\vec{p}$ and coordinate $\vec{r}$. Note that expression (9) can be used only on the tails of the energy distributions in the Boltzmann limit of an ideal gas, when $<n_{k}><<1$. Taking into account the motion of the matter, we replace the kinetic energy $\epsilon_{k}=E-m$ by $\epsilon_{k}=\gamma(E-\vec{p} \vec{v})-m$. The chemical potential included in formula (3) is redefined by $\mu=\mu_{T}+m$ in accordance with relativistic expressions. 


\section{References}

[1] H. Stoecker, W. Greiner, Phys. Rept. 137, 277 (1986)

[2] A.S. Khvorostukhin, V.D. Toneev, Phys. Part. Nucl. Lett. 14, 9 (2017)

[3] A.S. Khvorostukhin, V.D. Toneev, Phys. Atom. Nucl. 80, 285 (2017)

[4] A.T. D’yachenko, Phys. Atom. Nucl. 57, 1930 (1994)

[5] A.T. D’yachenko, K.A. Gridnev, W. Greiner, J. Phys. G. Nucl. Part. Phys. 40, 085101 (2013)

[6] A.T. D’yachenko, I.A. Mitropolsky, Bull. Russ. Acad. Sci. Phys. 80, 916 (2016)

[7] A.T. D’yachenko, I.A. Mitropolsky, Bull. Russ. Acad. Sci. Phys. 81, 1521 (2017)

[8] S.I. Gogolev et. al., in Proc. Int. School-Seminar on Heavy Ion Physics v.2, E7-93-274 (JINR, Dubna, 1993) 66

[9] B.M. Abramov et. al., Phys. Atom. Nucl. 78, 373 (2015)

[10] A.T. D'yachenko, I.A. Mitropolsky, Construction of the non-equilibrium equation of state for the description of collisions of heavy ions and its application to finding energy spectra of protons and subthreshold pions in the book "In Memory K. A. Gridnev. To the 80th anniversary of his birth" (Gatchina, NRC "Kurchatov Institute "- PNPI, 2018) 63. (in Russian)

[11] A.T. D'yachenko, V.N. Baryshnikov, in Proc. of Baldin ISHEPP XIX v.2 (JINR, Dubna, 2008) 234

[12] A.I. Anselm, Fundaments of statistical physics and thermodynamics (Moscow, Nauka, 1973) (in Russian)

[13] L.D. Landau, E.M. Lifshitz, Statistical Physics. Part 1 (Pergamon Press, 1985)

[14] V.V. Avdeichikov et al. (CHIC-Collaboration), in Proc. Int. School-Seminar on Heavy Ion Physics v. 2, E7-93-274 (JINR, Dubna, 1993) 238

[15] A.V. Dementyev, N.M. Sobolevsky, Nucl. Tracks Radiat. Meas. 30, 553 (1999)

[16] S.G. Mashnik et al., LA-UR-08-2931, arXiv:0805.0751 [nucl-th] (2008)

[17] T. Koi et al., AIP Conf. Proc. 896, 21 (2007)

[18] M.A. Braun, V.V. Vechernin, Sov. J. Nucl. Phys. 43, 1016 (1986) 